\title{
Impact of the Bundled End-Stage Renal Disease Payment System on Patient Care
}

\author{
Franklin W. Maddux \\ Fresenius Medical Care North America, Waltham, Mass., USA
}

\section{Key Words}

End-stage renal disease $\cdot$ Dialysis $\cdot$ Prospective payment system $\cdot$ Quality incentive program

\begin{abstract}
This article provides a perspective on the impact of the bundled end-stage renal disease prospective payment system on patient care. As the first year of the bundle comes to a close, we are beginning to see information about practice pattern changes that have resulted and the impact of those changes on the care of patients with renal disease. This review recognizes the background of the prospective payment system for renal replacement therapy and its evolution to include multiple components, as well as a description of the features of this payment system that are expected to have an impact on the clinical care by nephrologists and dialysis providers. Some of the expected impact on the dosing of erythropoiesis-stimulating agents, consolidation of dialysis providers, and a move toward more measurement are beginning to be seen. This brief summary not only reviews the components of the bundled payment system, but also the anticipated impact and some early information on the response to these predicted findings in the industry.
\end{abstract}

Copyright $\odot 2012$ S. Karger AG, Basel
(C) 2012 S. Karger AG, Basel

0253-5068/12/0333-0107\$38.00/0

Fax +41613061234 E-Mail karger@karger.ch www.karger.com

\section{Introduction}

The origins of the end-stage renal disease (ESRD) prospective payment system, the 'bundle,' began with a MedPAC report to the United States Congress in 2001 detailing the concepts of an adjusted payment system for ESRD care and renal replacement therapy by dialysis [1]. Enacted as part of the Medicare Improvements for Patients and Providers Act of 2008, the Centers for Medicare and Medicaid Services (CMS) was required to implement a new expanded bundled payment system for dialysis services by January 1, 2011 [2]. With implementation this past year, it is important to step back and look at the early perceptions and facts surrounding the impact of this adjusted value-based purchasing program on the provision of patient care for those with the most advanced renal disease. It is with detailed analysis of intended and unintended consequences of the payment system that we will learn most about how these adjustments have affected patients, nephrologists, and dialysis providers. These adjusted payment methodologies have fundamentally changed the renal industry now and for years to come. This perspective will review the components of the bundled payment system with respect to the impact on patients, the perceptions of the anticipated system, and the reality of what has actually occurred as a result of this new payment system. 


\section{Background}

The Medicare Improvements for Patients and Providers Act required CMS to implement an expanded bundled payment system for dialysis care by the beginning of 2011. These reforms adjusted the overall purchasing methodology that the CMS had developed since the initiation of the ESRD program in 1972, which in essence provided universal healthcare for patients with the most advanced renal disease in the United States [3]. The primary components of the bundled payment system included an overall reduction of $2 \%$ in costs for the provision of dialysis treatments and a removal of any potential for overuse of certain previously separately billable drugs such as erythropoiesis-stimulating agents (ESAs). Further, the development of the bundled payment system was the first disease state in which CMS determined clinical quality standards must be met to achieve full payment. Thus, the system represents the first global payment system consistent with the value-based purchasing philosophy that CMS has adopted as an effort to migrate healthcare payment from a traditional fee-for-service model toward one in which performance risk is imbedded in the payment system.

In addition, the bundle recognizes a desired migration toward patient centered healthcare where the ESRD patient sits at the center of a triangle made up of CMS as the federal payor, the nephrologist as the principle care giver, and the dialysis facility provider as the care implementation provider for patients undergoing dialytic therapies [4]. As such, this move toward a value-based purchasing model imbeds quality measurement into the fundamental fabric of the provision of care and invokes an expectation of continual measurement and adoption of evidencebased best clinical practices. The alignment of interests generated between the payor, physicians, and dialysis providers is a secondary goal desired by the federal government to optimize the health and improve longevity of patients with ESRD, provide unfettered access to treatment modality options for patients, and promote a triad of demonstrated quality dialysis care, improved patient safety, and more cost-efficient care.

\section{Impact of Payment Components}

The bundled ESRD payment system has a number of specific components that are changing how care is delivered to ESRD patients and offers an opportunity for novel systems and processes. Further, as the bundle was be- ing implemented, these payment components have generated a tremendous amount of industry discussion, debate, and controversy over the most appropriate use of certain medications, choices of therapy modalities, and the ability to capture certain patient conditions. The first surprise from the bundle was the adoption by almost all facilities to go 'all in' to the bundle and avoid a transition process that would phase in over 4 years. This was one of the first CMS assumptions that was not borne out in practice and was addressed in April 2011 by CMS to correct a transition financial penalty on facilities from the inaccurate estimation of facility participation.

\section{The Incident Patient}

The incident patient has received specific attention within the bundle due to a $51 \%$ increase in reimbursement for patients during their first 120 days of dialysis. Traditionally, the ESRD community has focused most measurement on the prevalent patient beyond the first 90 days of dialysis. In recent years, the United States Renal Data System (USRDS) has begun analysis of not only chronic kidney disease patients, but the impact of the transition period from chronic kidney disease to ESRD, looking at early mortality, hospitalization, and cost to the healthcare system for new dialysis patients. As such, the 120-day adjuster to the bundled payment system has driven heightened awareness demonstrated by increased publication, debate, and expectations for this patient cohort. Most dialysis providers have initiated programs to support heightened observation of incident patient health and to influence both the condition of the patient starting dialysis and methods to avoid hospitalization.

\section{Anemia Management}

With the bundled payment system, there are fundamental changes in the use of ESAs and iron which have led to the development by most dialysis providers and nephrologists of algorithms to manage the adjustments to ESAs and iron doses and reduce ad hoc dosing decisions. This has led to a reduction in the number of patients receiving ESAs and substantial discussion within the medical and provider community on how to measure and avoid both over- and underuse of medications for renal disease anemia management. Concurrent with changes to the care delivery under the bundle, there has been a growing body of evidence that high doses of ESA and high hemoglobin levels have consequent dangers to patients. This is a substantive divergence from prior guidance on ESA use and the inclusion of distinct hemoglobin levels as part of the Quality Incentive Program (QIP) under the bundle. In 
the middle of 2011, the FDA initiated a revision to the label on all ESA preparations that is expected to lower the acceptable levels of hemoglobin for patients with renal disease receiving ESAs and further restrict and diminish the use of these drugs. As such, the expected $15-18 \%$ reduction in ESA use that was anticipated prior to implementation of the bundle is expected to extend toward $20-30 \%$ reduction from peak levels of ESA utilization several years ago. These changes have brought about a degree of anxiety among patients, physicians, and providers in determining what optimal therapy for anemia of chronic kidney disease is today and concern over transfusion rates and the impact of low hemoglobin levels.

\section{Quality Incentive Program}

The QIP under the bundle has received a tremendous amount of comment as it is a complex system of measuring benchmark levels of attainment for dose of dialysis (urea reduction ratio $\geq 65 \%$ ) and anemia-related measures ( $\mathrm{Hgb}>12$, Hgb $<10$ for 2012) with subsequent reduction in payment to facilities during 2012 based on performance attained in 2010. In July of 2011, the QIP proposed rules for 2013 and 2014 were presented and made available for comment. This proposed set of additional elements has generated a vigorous debate about the disappearance of the QIP for low hemoglobin levels and the addition of vascular access, infection, and experience of care measures in 2014. At the time of this writing, there is extensive discussion regarding industry comments on the proposed QIP rule. Irrespective of the final 2014 rule, the QIP is expected to continue to be a fundamental driver of behavior by nephrologists and dialysis providers as it generates focus on specific elements of care for patients undergoing renal replacement therapy.

The ESRD PPS 2014 QIP proposed measure elements are:

- Continue Hgb $>12$ g/dl

- Adequacy: spKt/V $\geq 1.2$

- Vascular access

- Vascular access infection

- SHR admissions

- National Health Safety Network Dialysis Event

- Patient experience of care (ICH CAHPS)

- Mineral metabolism

\section{Comorbidities}

Comorbid disease has been one of the most difficult areas for implementation in the context of the bundle. The ability to identify and document selected acute and chronic comorbid conditions has been substantially ham- pered through lack of communication between the hospital and the dialysis facility to meet the verification standards for these comorbid conditions. The elements required to determine if the patient has had bacterial pneumonia, gastrointestinal bleeding, or pericarditis as an acute comorbidity and consistent validation of the myelodysplastic disorders and sickle disease represented by the chronic comorbid measures has been problematic. The actuarial rates expected in the bundle final rule has not been achieved in practice.

\section{Modality Selection}

The bundle payment system is expected to create a model where reimbursement and cost efficiencies might offer a wider array of therapeutic options for patients. As such, there have been a number of initiatives by CMS to encourage kidney disease education and broaden modality selection to enhance the volume of patients selecting a home therapy option. As such, one of the potential unintended consequences has been the recognition that for some patients this therapy has a limited life span due to the ongoing and distinct requirements on patients and their families that can lead to fatigue and 'modality churn'. During this first year of the payment system it may be too soon to have a complete understanding of these intended and unintended consequences, but it is clear that an expansion of home therapies for ESRD care is anticipated and appropriate with the appropriate selection of patients who may use these modalities as a long-term option.

\section{Outpatient Medications}

Home medication distribution and inclusion under the bundle is a major change in requirements for the dialysis facility. In anticipation of bringing all renal-related oral drugs into the bundle in 2014, dialysis providers have been required to engage in pharmacy services or to provide these medications to patients. This has been difficult and has led to a degree of confusion between pharmacies, dialysis providers, and patients as to which drugs are covered in the new payment system and the most efficient means of managing these prescribed medications. Also confusing is the inclusion of several additional items not originally understood by the renal industry as included in the bundle, such as topical anesthetics and certain catheter dressings. The concept of 'bundle integrity' has become a fashionable term to describe maintenance of the list of items included within the bundled payment process. As such, the drugs for renal disease care remain an ongoing topic of debate and evolution of the delivery model. The need to improve efficiency and consistency of 
managing these drugs, reconciling medications for patients, and looking for evidence-based treatment practices for bone and mineral metabolism, anemia management, and phosphorus reduction remains a challenge and opportunity for nephrologists and dialysis providers.

\section{Laboratory Studies}

Laboratory studies under the bundle are well defined with certain lab tests and frequencies being recognized. All other lab testing requires specific ICD-9 coding to support the physicians ordering choices and pattern. To date, the lab panels for ESRD have become reasonably well defined and, other than some confusion over particular lab tests or the inclusion of new tests that might be used to identify renal disease pathophysiology, are considered for ongoing discussion regarding the bundle. There has been a major change in the business models for ESRD-related laboratories as a result of the bundle.

\section{Conclusion}

The bundle as a novel payment system and methodology has engendered tremendous comment by the medical community and both the perception of the bundle prior to its initiation and the actual facts playing out during this first year of operation provide insight to the impact on patient care. There are several examples of discussion initiated before the implementation of the bundle include [5].

ASN president Joseph Bonventre, MD, articulated a concern for potential undertreatment with ESAs and dialysis time as the bundle was implemented. These concerns have generated active and ongoing discussion in the medical community about the value of extending dialysis time, especially in those patients with central venous catheters [6]. Further, with the change in ESA labeling from the FDA, there has been substantial turmoil in trying to determine the appropriate dosing strategies, effective hemoglobin levels, and capture of detailed information regarding patient transfusions given the limitations placed on dosing regimens for these anemia management drugs [7].

Prior to the bundle, Keith Johnson, MD, Chairman of Dialysis Clinic Inc., a nonprofit medium-sized dialysis organization, expressed concern about the potential for further consolidation of smaller and independent dialysis operators under the bundle. During the time of initiation of the bundle, additional consolidation had in fact begun through the acquisition of DSI by DaVita and the announced acquisition of the recently combined Liberty $\mathrm{Di}$ - alysis and Renal Advantage by Fresenius Medical Care. These consolidations may have coincided with the implementation of the bundle but, in speaking with principles at these organizations, are substantially directed towards positioning these organizations for a future within an integrated and accountable care framework for renal disease and maximizing the utilization of technologies to support more efficient care (F.W. Maddux, MD; personal communications, August 2011).

As CMS initiated the bundle, Administrator Donald Berwick, MD, recognized that the value-based purchasing program initiated by the QIP was a strong and initial step in fostering patient-centered care and a movement away from the traditional risk-free fee-for-service model that was driving higher costs and less than optimal quality of care. It remains to be seen if the patient-centered model of care is enhanced by the bundle. The Government Accountability Office has been charged with assessing access to care, medications, and modality choices for Medicare beneficiaries now in the ESRD program (Federal Register; PL: 111-148; Enacted 3/23/2010).

Further, the impact of information gathering and sharing on available information systems to account for comorbid disease identification, as well as assessment of performance and the science of measure development, places a greater need on the healthcare system for consolidated informatics and methods of developing an appropriate national renal-related health IT infrastructure. The expectations and demands for health information exchange with the federal government through CROWNWeb are enhanced by initiation of this payment system. The risks of a failed deployment of CROWNWeb are higher in the environment of the bundle, and it remains to be seen whether this program can be effectively deployed and sustained [8].

Finally, the ESRD prospective payment system has been initiated successfully with dialysis providers opting into the bundle at an unexpectedly high rate and the mechanics of payment under the bundle occurring relatively smoothly for most dialysis providers. The impact on clinical care and patients is substantial and clinical care has begun to change based on a number of drivers due to the bundle directly or in response to the adjusted ESA prescribing information articulated by the FDA. Nephrologists and their collaborating dialysis providers are more aligned today to organize the care of the patient along clinical pathways that will influence the delivery of care to not only those patients with a federal payor, but also to patients with commercial plans, state health plans, and other delivery systems. 


\section{References}

1 Medicare Payment Advisory Commission (MedPAC): Report to the Congress: Medicare payment policy, March 2001.

2 Centers for Medicare and Medicaid Services: End stage renal disease (ESRD) payment. http://www.cms.gov/ESRDPayment/ (accessed August 31, 2011).

3 Rettig R: Implementing the End Stage Renal Disease Program of Medicare (report). Arlington, RAND, 1980.
4 Lacson E, Hakim R: The 2011 ESRD prospective payment system: perspectives from Fresenius Medical Care, a large dialysis organization. Am J Kidney Dis 2011;57:547549.

5 Iglehart J: Bundled payment for ESRD - including ESAs in Medicare's dialysis package. N Engl J Med 2011;364:593-595.

6 Lacson E, Brunelli S: Hemodialysis treatment time: a fresh perspective. Clin J Am Soc Nephrol 2011;6:2522-2530.
7 Food and Drug Administration: Information on erythropoiesis-stimulating agents (ESA) epoetin alfa (marketed as Procrit, Epogen), darbepoetin alfa (marketed as Aranesp). http://www.fda.gov/drugs/drugsafety/postmarketdrugsafetyinformationforpatientsandproviders/ucm 109375.htm (accessed August 31, 2011).

$>8$ Delva O: How CROWNWeb will validate and utilize patient data. Nephrol News Issues 2010;24:30-33. 